\title{
Two Approaches to the Problem of High-cycle Fatigue of Materials and Structures
}

\author{
Vladimir Mironov ${ }^{1,2}$, Olga Lukashuk ${ }^{2, a}$ and Dmitriy Ogorelkov ${ }^{1,2}$ \\ ${ }^{1}$ Institute of Engineering Science, RAS (Ural Branch), 620049, 34 Komsomolskaya st., Yekaterinburg, Russia \\ ${ }^{2}$ Ural Federal University, 620002, 19 Mira Street, Yekaterinburg, Russia
}

\begin{abstract}
Creation of new types of materials and development of new methods aimed at extending the lifetime of structures are two interconnected approaches to solve the problem of metallic structures fatigue. The paper considers a method for estimating the efficiency of damping implemented in metallic structures by means of energy-intensive materials. The results of comparative calculation are given on the ultimate number of transverse vibration cycles in bridge girders. The calculation uses a linear hypothesis of fatigue-caused damage summation and a model of cyclic material degradation. Notable increase of the vibration decrement for foam-filled metallic structures is predicted, with the lifetime of products lengthening as a result.
\end{abstract}

\section{Introduction}

The problem of extending the lifetime of metallic structures of lifting and transporting machines is most often solved via technological techniques aimed at affecting the surface layer of metals or by means of diminishing variable dynamic loads. The latter method often uses structural damping [1-4]. The paper [5] discussed one of the approaches to increasing the durability of a thin-walled closedprofile beam by filling it with a low-modulus energy-intensive material. A method of evaluating the lifetime of structural elements which takes cyclic degradation of material properties into account was analyzed in [6,7]. This study uses that method for the purposes of comparatively estimating the lifetimes of unfilled and foam-filled bridge girders of an overhead crane.

The efficiency of structural damping applied to a metallic structure is assessed by running a costly experiment on the basis of a vibrorecord for free damped vibrations. Below a possibility is considered of theoretically predicting the lifetime of a filled bridge girder from a known logarithmic vibration decrement for unfilled beams. For example, the decrement $\delta_{1}$ for the span of a bridge crane in [8] is evaluated to be in the range of $\delta_{1} \in[0.05 ; 0.12]$. Thus, the decrement $\delta_{2}$ for filled beams could be found using the basic theory of vibrations for a material particle with its mass reduced to the centre of the span.

\section{Theoretical estimation of logarithmic decrement $\delta_{2}$}

\footnotetext{
${ }^{\text {a }}$ Corresponding author : oldim96@mail.ru
} 
Differential equation for damped vibrations of a material particle could be expressed as [9]

$$
\ddot{x}+2 n \dot{x}+p^{2} x=0
$$

where coefficient $n$ and circular frequency $p$ are linked to a circular frequency $p_{0}$, determined for free sustained (undamped) vibrations by the correlation $n^{2}=p_{0}^{2}-p^{2}$.

The decrement $\delta$, conditional period of vibrations $T$ and circular frequency are interconnected via the following expression:

$$
\delta=n T=n / p
$$

Denoting the vibration parameters for an unfilled girder by 1 in their lower indices and for a filled girder - by 2, and omitting insignificant effect of a light-weighted filler on the circular frequency $p_{0}$, the next two expressions could be formulated:

$$
n_{1}^{2}=p_{0}^{2}-p_{1}^{2} ; n_{2}^{2}=p_{0}^{2}-p_{2}^{2} \text {. }
$$

Then, elimination of $p_{0}$ gives us the expression $n_{2}=\sqrt{n_{1}^{2}+p_{1}^{2}-p_{2}^{2}}$, while taking Eq. 1 into account and allowing for the fact that a vibration frequency $\omega$ relates to the circular frequency as $p=2 \pi \omega$ helps us to obtain the desired expression in the form of

$$
\delta_{2}=\frac{1}{\omega_{2}} \sqrt{\left(\delta_{1} \omega_{1}\right)^{2}+\omega_{1}^{2}-\omega_{2}^{2}}
$$

The vibration frequencies $\omega_{1}$ and $\omega_{2}$ for unfilled and filled beams could easily be determined using a typical computer software, for example, such as SolidWorks [10,11]. When the decrement $\delta_{1}$ is pre-determined, Eq. 3 is completely defined.

The solution of Eq. 1 for a known coefficient $n$ provides the law of variation in point-mass displacements, and, for a linear system, even the law of variation in damped-vibration stresses

$$
\sigma=\sigma_{0} \exp (-n t)
$$

where $\sigma_{0}$ is a dynamic stress related, for example, to the acceleration applied to or removed from the system when starting to lift the load or applying the brakes. Constraining the level of significant amplitudes to the fatigue limit $\sigma_{R}$, the period of their action $t_{*}$ could be found on the condition of $\sigma_{0} \exp \left(-n t_{*}\right)=\sigma_{R}$. Then, the number of damped vibrations caused by a single dynamic load which produces the effect of $\sigma_{0}$ stress in a critical point of a metallic structure would be expressed as

$$
N_{Z}=\frac{t_{*}}{T}=\frac{-\ln \left(\sigma_{R} / \sigma_{0}\right)}{T n}=\frac{-\ln \left(\sigma_{R} / \sigma_{0}\right)}{\delta}
$$

In engineering calculations, $\sigma_{0}$ means the normal stress in a critical section of a beam $[8,12]$ or the von Mises stress [13]. The latter is determined in terms of high-cycle fatigue by solving a boundary value problem from the theory of elasticity. The SolidWorks software package provides desired numerical solution using the finite-element method.

\section{Model of material accounting for cyclic degradation}

For a specified law of variation in alternating loads, the durability could easily be predicted on the basis of a linear hypothesis of fatigue-caused damage summation [14]

$$
\sum \frac{n}{N}=1
$$


But, neither the measure of damage $\mathrm{n} / \mathrm{N}$, nor the criterion of failure (equaling 1 ) do not have any physical explanation. Correcting the right part from experimental data does not guarantee to have the accuracy of prediction maintained when the conditions of testing change. The authors base their approach to the problem of metal fatigue [7] on the experiments aimed at degrading such parameters of stress-strain diagrams as ultimate strength and ultimate strain. With a force approach chosen to describe the fatigue process and an exponential function picked to approximate an experiment-based kinetic curve, this expression follows:

$$
\mathrm{S}_{\mathrm{B}}\left(\sigma_{M} ; \mathrm{n}\right)=S_{B 0}-k_{\sigma} n^{m}
$$

where $S_{B 0}$ is ultimate strength, $\sigma_{M}$ is maximal stress in a reduced cycle, and the expression $k_{\sigma} n^{m}$ has been taken in the form of a Corten-Dolan damage function, which has its own experimental verification. Then, $k_{\sigma}$ could be determined on the condition of failure

$$
S_{B}\left(\sigma_{M} ; N_{M}\right)=\sigma_{M}
$$

playing the role of the criterion for the fatigue failure in the case of regular cyclic loading with the expression of Eq. 7 taking the form of

$$
S_{B}\left(\sigma_{M} ; n\right)=S_{B 0}-\frac{S_{B 0}-\sigma_{M}}{N_{M}^{m}} n^{m},
$$

where $N_{M}$ is the durability by the Weller diagram, $\mathrm{m}$ is an experimental material constant.

Generalization of the approach onto nonstationary load is defined on the condition of equivalence of two cyclic material states with a different history of loading:

$$
S_{B}\left(\sigma_{1} ; n_{1}\right)=S_{B}\left(\sigma_{2} ; n_{2}\right)
$$

This equation helps to find an equivalent (in terms of damage) number of cycles $n_{\ni}$ in that kinetic curve to which the transition is made. The criterion of fatigue failure in Eq. 8 is generalized onto the nonstationary load in the form of a rule for intersection of an actual material strength and the level of maximal stress in the cycle.

How the lack of uniaxiality in the stressed state affects the lifetime has not been studied in a sufficient detail. It is known, for example, that the second main stress did not impact the lifetimes of pipe specimens if it was less than 0.8 of the main stress itself [15]. In that case, the kinetic curve from Eq. 7 could be used immediately in phenomenological calculation.

Eqs. 7-10 form the original part of the model of material under cyclic loading and fit known expressions of the classic continual theory of fatigue. This fact allows to include into the model experimentally-verified results of long-term studies on the topic of materials and structures fatigue strength. For example, classification of components in terms of stress concentration and a table of basic strength limits $\sigma_{-1 K B}$ factoring in the quality class for welded seams [16] certainly would be useful when designing welded metallic structures.

An important correction into the procedure of evaluating the ultimate strength $\sigma_{-1 K}$ for a $t$-thick element is introduced by the scale effect

$$
\sigma_{-1 K}=\sigma_{-1 K B}\left(\frac{t_{B}}{t}\right)^{0,2}
$$

where $t_{B}$ is thickness of a basic specimen.

The fatigue curve could be used both in its typical form

$$
\sigma_{R}^{\alpha} N_{B}=\sigma_{i}^{\alpha} N_{i}
$$

and in any other form derived from the results of mechanical testing on the material fatigue. On the other hand, the non-linear function of Eq. 7 presumes that the damaging action of the cycle of stresses depends on where they are located in the whole spectrum of loading. Therefore, regrouping of cycles 
- which is a common practice in correlation and spectrum analyses of loading - is excluded. To illustrate the point, self-induced transverse vibrations of bridge girders of an overhead crane should be considered. Those emerge at the moments of starting and stopping the travelling mechanism and come in two variants - for unfilled and foam-filled girders. The symmetry in the cycle of loading (with a constant asymmetry coefficient $R=-1$ ) is provided by varying stresses in accordance with the law of Eq. 4.

\section{Vibrations of bridge girders of overhead cranes}

The dimensions of a bridge girder for an overhead crane of $19.5 \mathrm{~m}$ in span and $20 t$ in lifting capacity were found in typical strength and rigidity calculations. That beam is $1.1 \mathrm{~m}$ high and $0.6 \mathrm{~m}$ wide, and the required thickness of its wall is $9 \mathrm{~mm}$. A finite-element model of an unfilled beam was built in SolidWorks (Fig. 1), followed by an assembly model with a filler.

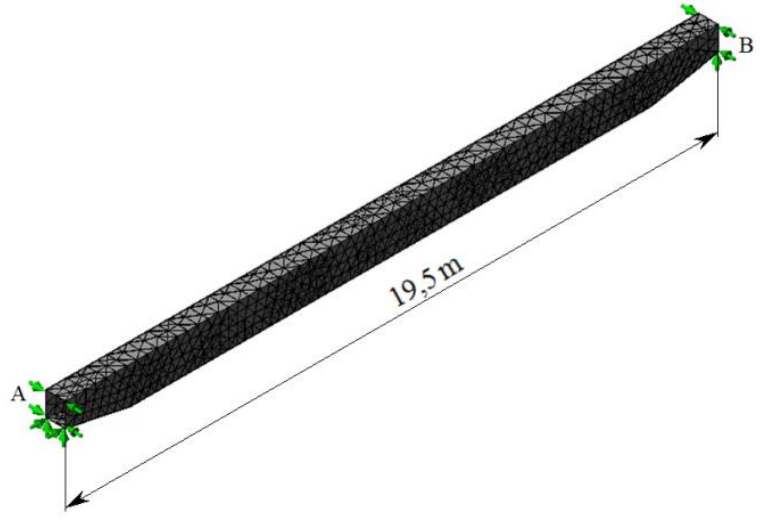

Figure 1. Finite-element model of bridge girder.

Foam was chosen to act as a filler, having the following characteristics: modulus of elasticity -0.5 $M P a$, Poisson coefficient -0.49 , mass density $-10 \mathrm{~kg} / \mathrm{m}^{3}$. Lower frequencies in the horizontal plane were obtained as $\omega_{1}=7.0546 s^{-1} ; \omega_{2}=6.980 s^{-1}$. The efficiency of structural damping implemented in a beam could be evaluated on the logarithmic decrement $\delta_{2}$, which is calculated using Eq. 5 for various values of the logarithmic decrement $\delta_{1}$ for an unfilled beam (Fig. 2).

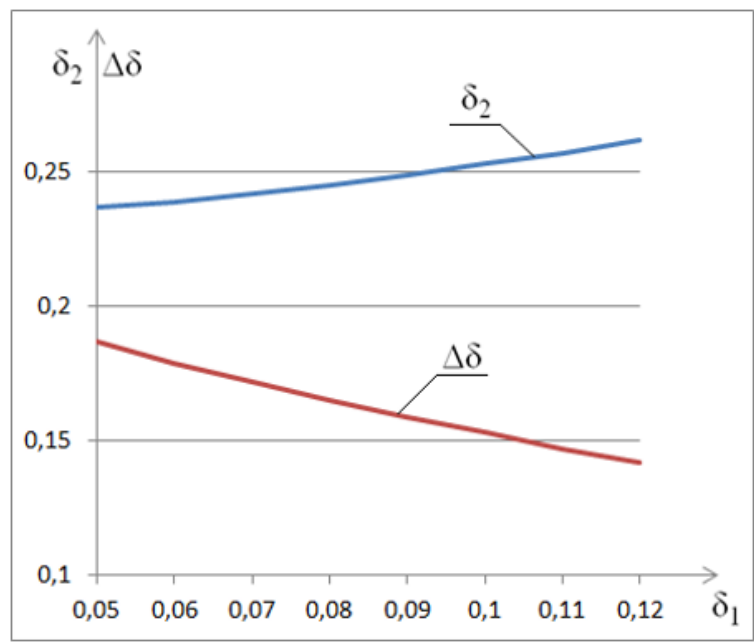

Figure 2. Efficiency of structural damping. 
If the efficiency is estimated on the expression $\Delta \delta=\delta_{2}-\delta_{1}$, one should note that it decreases with the increase of $\delta_{1}$. The graphs above clearly illustrate that damping bridge girders by means of foam materials is certainly quite efficient. The effect also shows up in the number of significant vibration amplitudes $N_{z}$ in the case of a one-time application of dynamic load. For example, the number dropped from 18 to 6 vibrations for $\delta_{1}=0.05$. It could be demonstrated that the dampingcaused decrease of the amplitude and the number of self-induced vibration cycles $N_{z}$ (determined by Eq. 3) leads to the extension of the product lifetime.

The loads for starting and braking operational modes of the crane were evaluated using a dynamic model of the beam travelling alongside the trolley and load itself. That evaluation provided the following results: maximal normal stresses in the beam when the crane stopped $-\sigma_{0}=120 \mathrm{MPa}$; constrained fatigue limit for $N_{B}=2 \cdot 10^{6}$ cycles, welded seams and the scale effect of Eq. $11-\sigma_{R}$ $=50 \mathrm{MPa}$; indicator of the fatigue curve from Eq. 12 for the St3sp3 steel $-\alpha=5,34$ [17].

When the maximal stresses in the cycle change following the law of Eq. 4, a cycle-by-cycle alteration of stresses occurs. One block of such stresses consists of $N_{z}$ damped-amplitude cycles. And for the $i$-th cycle, the following expression is true:

$$
\sigma_{i}=\sigma_{0} e^{-n \mathrm{Ti}}=\sigma_{0} e^{-\delta i}
$$

When Eqs. 8 and 9 are considered together, it leads to the expression which allows to calculate an actual value of the ultimate strength after a certain equivalent number of cycles

$$
S\left(\sigma_{M 1}, n_{E}+\Delta n_{1}\right)=S_{B 0}-\frac{S_{B 0}-\sigma_{M 1}}{N_{M 1}^{m}}\left(n_{E}+\Delta n_{1}\right)^{m},
$$

where $\Delta n=1$ for the cycle-by-cycle variation in stress amplitudes (as in the example).

Based on this equation, an algorithm was developed for comparative calculation of the number of cycles prior to the failure of a metallic structure due to transverse vibrations. The algorithm uses the linear hypothesis of Eq. 6 and the model of cyclic material degradation described. For a number of steels, a numerical value of the constant $\mathrm{m}$ was close to 2 , and, therefore, the calculation assumed $\mathrm{m}=$ 2. The numbers of cycles prior to the failure due to transverse vibrations for the linear damage summation NLH and in terms of the proposed model of material ND (Table 1) are expressed in blocks of vibrations caused by a single dynamic load.

Table 1. Results of computing experiments.

\begin{tabular}{|c|c|c|c|}
\hline \multicolumn{2}{|c|}{ Decrement } & $N_{L H}$ & $N_{D}$ \\
\hline$\delta_{1}$ & $0.05 \div 0,12$ & $5716 \div 16514$ & $4302 \div 8692$ \\
\hline$\delta_{2}$ & $0.155 \div 0,19$ & $23510 \div 31743$ & $10364 \div 11528$ \\
\hline
\end{tabular}

The table shows the interval boundaries of variation in the logarithmic decrements $\delta_{1}$ and $\delta_{2}$ (calculated using Eq. 3). The second column provides lifetimes in terms of cycle blocks calculated using the linear hypothesis for the specified boundary values of the decrements in the first column. Those numbers indicate that the structural damping affects notably the lifetimes. The third column gives lifetimes for the same decrements calculated using the model which allows for the cyclic degradation of material. A substantial discrepancy is observed between the predictions of the ultimate 
number of cycles, and it is especially the case with smaller values of the decrement $\delta_{1}$ for unfilled structures.

\section{Summary}

1. The approach is given to estimating the logarithmic decrement for metallic structures filled with an energy-intensive material on the basis of a pre-determined decrement found in literature sources.

2. Large increase of the decrement is predicted when a foam material is used as a light-weighted filler for metallic structures with closed-profile cross-sections. This relatively simple and reliable type of structural damping could prove to be quite competitive in terms of comparison with other appliances meant to damp vibrations (such as electromechanical or hydromechanical systems).

3. Fast damping of vibrations is vital for many designs, including those where precise positioning is important, and, in particular, in the case of bridge girders of an overhead crane. The calculated number of significant self-induced vibrations of such girders decreases by two or three times, which greatly affects the lifetimes of their metallic structures.

4. The prediction of the ultimate number of cycles for transverse vibrations using a model of cyclic material degradation substantially differs from the prediction which uses a linear hypothesis of fatigue damage summation, with shorter lifetimes predicted by the model.

\section{References}

1. V.N. Kobelev, L.M. Kovarskiy, S.I. Timofeev, Design of Three-Layer Structures (Mashinostroenie, Moscow, 1984)

2. K.V. Frolov (Ed.), Protection from Vibrations and Impacts (Mashinostroenie, Moscow, 1981)

3. Singiresu S. Rao, Mechanical Vibrations (Addison-Wesley Pung Company, 1995)

4. On-line ANSYS Theory Reference, Release 9.0

5. V.I. Mironov, O.A. Lukashuk, D.A. Ogorelkov, Proceedings of MATEC Web Conference (2017)

6. V.I. Mironov, O.A. Lukashuk, D.I. Vichuzhanin, J. Sol. St. Phen., 815, 815-820 (2017)

7. I.G. Emel'yanov, V.I. Mironov, Durability of Shell Structures (UrO RAN Publishing, Yekaterinburg, 2012)

8. S.A. Sokolov, Structural Mechanics and Metallic Structures of Machines (Politehnika, SaintPetersburg, 2011)

9. Y.G. Panovko, Basics of Applied Theory of Elastic Vibrations (State Science and Technology Publisher of Engineering Literature, Moscow, 1957)

10. A.A. Alyamovskiy, A.A. Sobachkin, E.V. Odintsova, et al., Computer-aided Design in SolidWorks for Engineering Purposes (BHV-Peterburg, Saint Petersburg, 2005)

11. K.A. Basov, ANSYS. User's Manual (DMK-Press Publ., Moscow, 2005)

12. Standard for calculationand designing of cars of railways of Ministry of Railways of a track of $1520 \mathrm{~mm}$ (not automotive)., (GosNIIV-VNIIZhT, Moscow, 1996)

13. Godunov S.K., J. Russian Mathematical Surveys, vol.16, 3, 171 - 174 (1961)

14. A. Miner, J. Appl. Mech., 12, 159-164 (1945)

15. N.I. Grinenko, Proceedings of School-Seminar on Fatigue Strength of Machine-Building Structures (1975)

16. V.I. Trufyakov, Fatigue of Welded Joints (Naukova dumka, Kiev, 1973)

17. V.T. Troshhenko, L.A. Sosnovskiy, Fatigue Strength of Metals and Alloys (Naukova Dumka, Kiev, 1987) 